\title{
Influence of quantum well inhomogeneities on absorption, spontaneous emission, photoluminescence decay time, and lasing in polar InGaN quantum wells emitting in the blue-green spectral region
}

\author{
M. Gladysiewicz $\cdot$ R. Kudrawiec $\cdot$ M. Syperek • \\ J. Misiewicz • M. Siekacz • G. Cywinski • \\ A. Khachapuridze · T. Suski • C. Skierbiszewski \\ Received: 16 February 2013 / Accepted: 14 August 2013 / Published online: 5 September 2013 \\ (C) The Author(s) 2013. This article is published with open access at Springerlink.com
}

\begin{abstract}
It is shown that in polar InGaN QWs emitting in the blue-green spectral region a Stokes shift between spontaneous emission (SE) and optical transition observed in contactless electroreflectance (CER) spectrum (absorptionlike technique) can be observed even at room temperature, despite the fact that the SE is not associated with localized states. Time resolved photoluminescence measurements clearly confirm that the SE is strongly localized at low temperatures whereas at room temperature the carrier localization disappears and the SE can be attributed to the fundamental transition in this QW. The Stokes shift is observed in this QW system because of the large built-in electric field, i.e., the CER transition is a superposition of all optical transitions with non-zero electron-hole overlap integrals and, therefore, the energy of this transition does not correspond to the fundamental transition of InGaN QW. Lasing from this QW has been observed at the wavelength of $475 \mathrm{~nm}$, whereas the SE was observed at $500 \mathrm{~nm}$. The $25 \mathrm{~nm}$ shift between the lasing and SE is observed because of a screening of the built-in electric field by photogenerated carriers. However, our analysis shows that the built-in electric field inside the InGaN QW region is not fully screened under the lasing conditions.
\end{abstract}

\footnotetext{
M. Gladysiewicz $(\bowtie) \cdot$ R. Kudrawiec $\cdot$ M. Syperek · J. Misiewicz Institute of Physics, Wrocław University of Technology, Wybrzeże Wyspiańskiego 27, 50-370 Wrocław, Poland e-mail: marta.gladysiewicz@pwr.wroc.pl

M. Siekacz · G. Cywinski · A. Khachapuridze · T. Suski · C. Skierbiszewski Institute of High Pressure Physics, Polish Academy of Sciences, Sokołowska 29/37, 01-142 Warsaw, Poland
}

\section{Introduction}

Wurtzite InGaN quantum wells (QWs) are a very important gain medium for fabricating optoelectronic devices, such as near ultraviolet, blue, and green light emitting diodes (LEDs) and laser diodes (LDs) [1-14]. The growth of InGaN QWs along the [0001] direction leads to a QW system with a very strong quantum confined Stark effect due to the large internal electric fields which result from discontinuities in spontaneous and piezoelectric polarization at $\mathrm{QW}$ heterointerfaces $[15,16]$. These fields cause spatial separation of electrons and holes and reduce the carrier recombination efficiency. This issue is especially important for green InGaN LEDs and LDs because of higher indium concentrations, i.e., larger strains and hence stronger piezoelectric fields incorporated in these devices [14]. In addition to the strong built-in electric field, other effects like (i) indium content fluctuations including a formation of indium-rich areas similar to quantum dots, (ii) generation of native point defects and threading dislocations are expected to be crucial for InGaN QWs dedicated for the green spectral range [14]. An accurate diagnostic of each effect seems to be necessary for the optimization of growth conditions and the improvement of LEDs and LDs performances. Usually, such a diagnostic cannot be limited to an ordinary photoluminescence because of difficulties in distinguishing between localized emission (unpromising emission for LD applications) and delocalized emission (promising emission for LD applications). Better understanding of the emission mechanism and the relation between the wavelength of spontaneous emission and lasing form polar InGaN QWs is expected to help in the future development of blue-green LEDs and LDs. In this context a deeper interpretation of emission spectra, its temporal dynamics and correlation with absorption-like measurements, and theoretical calculations for various built-in 
electric fields in InGaN QW system are necessary. The aim of this paper is to provide such an interpretation for $\mathrm{InGaN}$ QW structures emitting in the blue-green spectral range.

\section{Experimental details}

InGaN QW structures were grown by plasma assisted molecular beam epitaxy (MBE) in an Oxford VG90 system under In-rich conditions on free standing Ga-polar bulk GaN substrates. The system was equipped with two Veeco RF plasma sources operating at $240-450 \mathrm{~W}$ for $0.8-2 \mathrm{~cm}^{3} / \mathrm{min}$ of $\mathrm{N}_{2}$ flow. The pressure during growth was $1.5-7 \times 10^{-5}$ Torr. Relevant details on the sample growth can be found in Ref. [13]. In this work we focus on the sample with the active part composed of three $3 \mathrm{~nm}$ wide $\mathrm{In}_{0.25} \mathrm{Ga}_{0.75} \mathrm{~N}$ QWs separated by $10 \mathrm{~nm}$ thick $\mathrm{In}_{0.06} \mathrm{Ga}_{0.94} \mathrm{~N}$ barriers slightly doped by silicon ([Si] $\sim 10^{17} \mathrm{~cm}^{-3}$ ).

The optical properties of InGaN QW structures were investigated by contactless electroreflectance (CER), standard photoluminescence (PL), and time resolved PL (TRPL). For the study of optical lasing the $\mathrm{Al}_{0.08} \mathrm{Ga}_{0.92} \mathrm{~N}$ cladding were grown in order to confine the optical mode [13]. PL and TRPL measurements were performed for the same sample as lasing measurements. For CER measurements InGaN QW samples without the top cladding layer were grown since no CER signal from the QW region was observed for samples with the top cladding due to small penetration depth of CER technique [17].

For CER measurements samples were mounted in a capacitor with the top electrode made from a copper-wire mesh which is semi-transparent for light. This electrode was kept at a distance of $\sim 0.5 \mathrm{~mm}$ from the sample surface while the sample itself was fixed on the bottom copper electrode. A maximum peak-to-peak alternating voltage of $\sim 3.5 \mathrm{kV}$ with the frequency of $285 \mathrm{~Hz}$ was applied. The sample was illuminated by spectrum of white light from a halogen lamp $(150 \mathrm{~W})$ at normal incidence. The light reflected from the sample was dispersed through a $0.55 \mathrm{~m}$ focal length single grating monochromator and detected by a photomultiplier and $\mathrm{Si}$ photodiode in the range of QW transitions. Other relevant details of CER measurements are described in Refs. $[18,19]$. To measure PL spectra at low excitation conditions the sample was excited by a $375 \mathrm{~nm}$ line of a continuous wave laser. The excitation intensity was $1 \mathrm{~mW}$ and the laser beam was focused to the diameter of $\sim 0.2 \mathrm{~mm}$.

For the TRPL measurements, samples were excited by a mode-locked Ti:Sapphire laser with $76 \mathrm{MHz}$ repetition rate and the pulse duration of $150 \mathrm{fs}$. Frequency-doubled pulses of the wavelength of $380 \mathrm{~nm}$ were used to excite the samples. In order to investigate the influence of point defects on dynamics of photoluminescence, TRPL measurements were performed at low/medium excitation conditions. The average excitation intensity was $25 \mu \mathrm{W}$ and the laser beam was focused to the diameter of $\sim 0.2 \mathrm{~mm}$. The PL signal was dispersed by a $0.3 \mathrm{~m}$-focal length monochromator and detected by a streak camera with $\mathrm{S} 1$ cathode. For low temperature TRPL measurements the samples were mounted in a helium flow cryostat.

For measurements of the optical lasing the samples were cleaved for thick bars of the length $L=0.4-1.2 \mathrm{~mm}$. Such a bar was excited by the third harmonics $(355 \mathrm{~nm})$ of Nd:YAG laser beam with the aperture size of $0.25 \mathrm{~mm}$, see Ref. [13] for details of experimental configuration. The laser pulses had the duration of $5 \mathrm{~ns}$ and the repetition rate of $20 \mathrm{~Hz}$. In order to determine the threshold excitation intensity for lasing, the measurements were performed for various excitation intensities up to the maximal intensity of $20 \mu \mathrm{J}$.

\section{Results and discussion}

Figure 1(a) and (b) show PL and CER spectrum, respectively, measured at room temperature for the InGaN QW structure. We can assume that the built-in electric field is not screened during PL measurements since the excitation intensity is weak $\left(\sim 2 \mathrm{~W} / \mathrm{cm}^{2}\right)$. It is clearly visible that the peak position of spontaneous emission (SE) does not correspond to the optical transition in CER spectrum which is insensitive to localized states because of its absorption-like character (see the dashed vertical lines in Fig. 1). Usually the difference between the energy of SE and CER transition (i.e., the Stokes shift) is evidence of the localized character of SE [20-23]. However, such a comparison is more complex for polar QWs due to the large built-in electric field related to polarization effects. In this structure the electronhole overlap for the optical transition between the first-hole and the first-electron level (1h-1e transition) can be smaller than the overlap for the transition between the first-hole and the second-electron level (i.e., the $1 \mathrm{~h}-2 \mathrm{e}$ transition, which is nominally forbidden for squarelike QWs without built-in electric field). It means that the broad CER transition can be a superposition of the optical transitions between the ground and excited states in the InGaN QW (i.e., the strongest CER signal does not correspond to the fundamental transition in the InGaN QW). In contrast to CER, the main contribution to PL spectrum origins from the ground state transition even while the electron-hole overlap integral for this transition is smaller than for optical transitions between excited states (e.g. $2 \mathrm{e}-1 \mathrm{~h}$ or $1 \mathrm{e}-2 \mathrm{~h}$ transition). Finally the lasing measurements confirm the conclusion that the strongest CER signal does not correspond to the ground state transition in polar InGaN QW, see in Fig. 1(a) that the lasing peak is observed $\sim 20 \mathrm{~nm}$ below the maximal CER signal which is related to QW region. It is worth noting that the lasing peak cannot be attributed to an excited state since the energy separation the ground and excited states is large for such a narrow QW, 


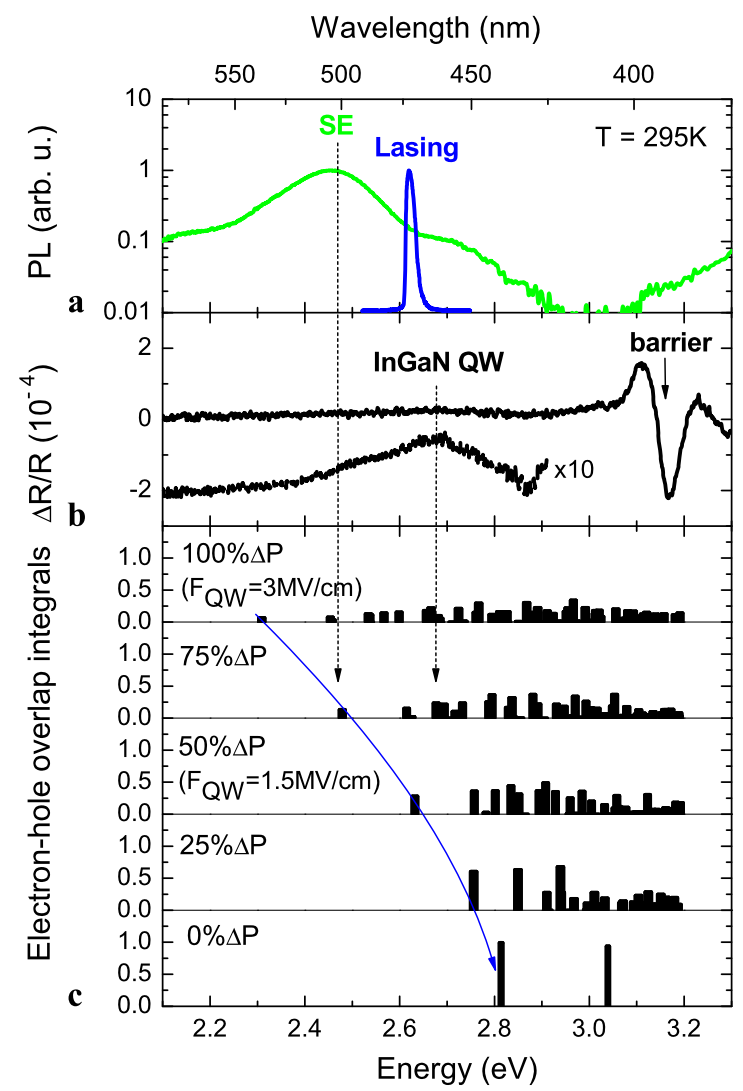

Fig. 1 (a) Photoluminescence (green line) and lasing (blue line) from InGaN QW structure at room temperature. (b) Room temperature contactless electroreflectance spectrum of InGaN QW structure without the top cladding layer (in the range of QW transitions the contactless electroreflectance spectrum has been measured with better signal to noise ratio, multiplied by the factor of ten, and shifted for better visualization). (c) Electron-hole overlap integrals for optical transitions in InGaN QWs of various polarization degrees $\Delta P$, which corresponds to various built-in electric fields $\left(F_{\mathrm{QW}}\right)$ in the $\mathrm{QW}$ region

as shown in the next part of this paper, and the electronhole overlap for $2 \mathrm{e}-1 \mathrm{~h}$ and $2 \mathrm{~h}-1 \mathrm{e}$ transitions decreases very significantly when the QW is strongly populated by carriers since the built-in electric field is screened. It means that the ground state transition is located at lower energy than the maximal CER signal.

In order to support the foregoing explanation of the broad CER transition and the large Stokes shift, the energy level structure and electron-hole overlaps for all possible optical transitions in this QW system have been calculated in the framework of the electron effective mass approximation, see details of calculations in Ref. [24]. The material parameters for these calculations were taken from Ref. [25] and they are given in Table 1. For $\operatorname{In}_{x} \mathrm{Ga}_{1-x} \mathrm{~N}$ all these parameters except the energy gap were calculated according to Vegard's law:

$\alpha_{\mathrm{InGaN}}=x \alpha_{\mathrm{InN}}+(1-x) \alpha_{\mathrm{GaN}}$,
Table 1 Material parameters for $\mathrm{InN}$ and $\mathrm{GaN}$

\begin{tabular}{lcc}
\hline Parameter $^{\mathrm{a}}$ & $\mathrm{InN}$ & $\mathrm{GaN}$ \\
\hline Energy gap at $295 \mathrm{~K}(\mathrm{eV})$ & 0.65 & 3.44 \\
$m_{e}\left(m_{0}\right)$ & 0.07 & 0.20 \\
$m_{h}(\mathrm{~A}$ band $)\left(m_{0}\right)$ & 1.0 & 0.85 \\
$a_{t}^{c}(\mathrm{eV})$ & -3.5 & -4.9 \\
$a_{z}^{c}(\mathrm{eV})$ & -3.5 & -11.3 \\
$D_{1}(\mathrm{eV})$ & -3.7 & -3.7 \\
$D_{2}(\mathrm{eV})$ & 4.5 & 4.5 \\
$D_{3}(\mathrm{eV})$ & 8.2 & 8.2 \\
$D_{4}(\mathrm{eV})$ & -4.1 & -4.1 \\
$c_{31}(\mathrm{GPa})$ & 92 & 106 \\
$c_{33}(\mathrm{GPa})$ & 224 & 398 \\
$e_{31}\left(\mathrm{C} / \mathrm{m}^{2}\right)$ & -0.45 & -0.37 \\
$e_{33}\left(\mathrm{C} / \mathrm{m}^{2}\right)$ & 0.81 & 0.67 \\
$P^{S}\left(\mathrm{C} / \mathrm{m}^{2}\right)$ & -0.042 & -0.034 \\
$\varepsilon\left(\varepsilon_{0}\right)$ & 15 & 9.0 \\
$a(\AA)$ & 3.545 & 3.189 \\
$c(\AA)$ & 5.703 & 5.185 \\
\hline
\end{tabular}

${ }^{\text {a } R e f . ~[25] ~}$

where $\alpha_{\mathrm{InN}}$ and $\alpha_{\mathrm{GaN}}$ are parameters of binary materials $\left(D_{1}, D_{2}\right.$, etc.). The energy gap for InGaN was calculated using the following formula:

$E_{g}^{\mathrm{InGaN}}=x E_{g}^{\mathrm{InN}}+(1-x) E_{g}^{\mathrm{GaN}}-c x(1-x)$,

with the bowing parameter $c$ assumed to be $1.7 \mathrm{eV}$ according to our recent investigations for InGaN alloys with 17-36\% indium [26]. The strain-related shifts of the valence and conduction band $\left(\delta E_{C B}\right.$ and $\left.\delta E_{A}\right)$ in InGaN layer were calculated according to the following expressions [27]:

$\delta E_{c}=\left(a_{z}^{c}-a_{t}^{c} \frac{c_{33}}{c_{31}}\right) \varepsilon_{z}$,

$\delta E_{A}=\left(D_{1}+D_{3}-\left(D_{2}+D_{4}\right) \frac{c_{33}}{c_{31}}\right) \varepsilon_{z}$,

where $c_{31}$ and $c_{33}$ are the elastic stiffness constants, $D_{1}, D_{2}$, $D_{3}$, and $D_{4}$ are valence band deformation potentials, and $a_{t}^{c}$ and $a_{z}^{c}$ are the hydrostatic deformation potentials. $\varepsilon_{z}$ is the strain which is defined as $\varepsilon_{z}=\left(c-c_{0}\right) / c_{0}$, where $c$ is the lattice constant of the free-strained crystal (InGaN in this case) and $c_{0}$ is the lattice constant of the substrate $(\mathrm{GaN})$. The electric field in the InGaN QW and GaN barriers was calculated according to the periodic boundary conditions [28] of Eq. (4):

$F_{n}=\frac{\sum_{q} \frac{l_{q} P_{q}}{\varepsilon_{q}}-P_{n} \sum_{q} \frac{l_{q}}{\varepsilon_{q}}}{\sum_{q} \frac{l_{q}}{\varepsilon_{q}}}$,

where the sum runs over all the layers. The $\varepsilon_{q}, P_{q}$, and $l_{q}$ is the dielectric constant, total polarization, and width of 


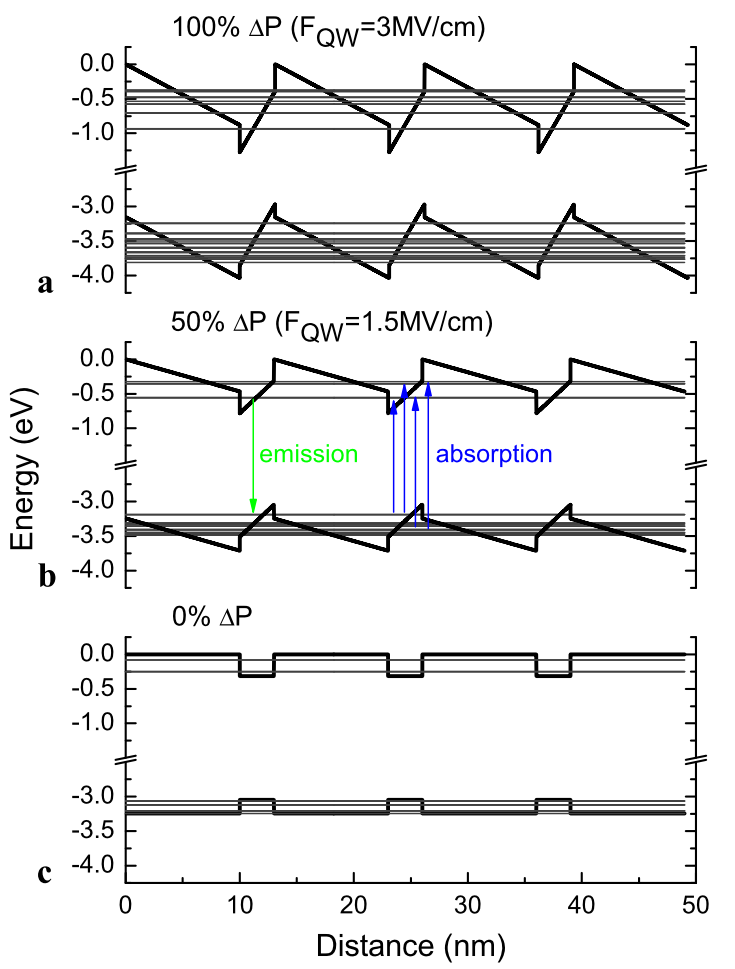

Fig. 2 Conduction and valence band confinement potential with the calculated electron and hole levels for InGaN QW structure of various polarization degrees $\Delta P$, which corresponds to various built-in electric fields

the $q$ th layer, respectively. The total polarization is given by Eq. (5):

$P_{n}=P_{n}^{S}+P_{n}^{P}$,

where $P_{n}^{S}$ and $P_{n}^{P}$ is the spontaneous and piezoelectric polarization in $n$th layer, respectively. Spontaneous polarization in $\operatorname{In}_{x} \mathrm{Ga}_{1-x} \mathrm{~N}$ alloy was calculated according to Eq. (6)

$P_{\mathrm{InGaN}}^{S}=x P_{\mathrm{InN}}^{S}+(1-x) P_{\mathrm{GaN}}^{S}+b x(1-x)$,

where $b$ is the bowing parameter for the spontaneous polarization in $\operatorname{In}_{x} \mathrm{Ga}_{1-x} \mathrm{~N}$, which is assumed to be $-0.037 \mathrm{C} / \mathrm{m}^{2}$ after Ref. [25]. The piezoelectric polarization was calculated using Eq. (7):

$P_{\mathrm{InGaN}}^{P}=\left(e_{31}-e_{33} \frac{c_{13}}{c_{33}}\right)\left(\varepsilon_{x}+\varepsilon_{y}\right)$,

where $\varepsilon_{x}$ and $\varepsilon_{y}$ are strains which are defined as $\varepsilon_{x}=\varepsilon_{y}=$ $\left(a-a_{0}\right) / a_{0}$, where $a$ is the lattice constant of the freestrained crystal (InGaN in this case) and $a_{0}$ is the lattice constant of the substrate $(\mathrm{GaN})$. The piezoelectric tensor components $\left(e_{31}\right.$ and $\left.e_{33}\right)$ and the elastic stiffness constants $\left(c_{13}\right.$ and $c_{33}$ ) were calculated for InGaN according to Vegard's law given by Eq. (1).

Figure 2 shows the results of our calculations for the InGaN QW sample for a few situations. The first situation [Fig. 2(a)] was obtained at the assumption that the built-in electric field inside the QW system results from the piezoelectric and spontaneous polarizations in this system $[15,16]$ with the appropriate periodic boundary conditions [28] (this situation is characterized by a step-like difference in the total polarization at $\mathrm{QW}$ interfaces $\Delta P$ ). It should be noted that the internal electric field, which results from polarization effects, can be partially screened in our samples because of Si doping in InGaN barriers [29-31]. It means that the real built-in electric field is smaller than the electric field corresponding to $100 \% \Delta P$ in Fig. 2(a) and, therefore, additional situations (i.e., other $\Delta P$ values) have been considered, see Fig. 2(b) and (c). In addition, appropriate diagrams of optical transitions in InGaN QW system are shown in Fig. 1(c). These diagrams shows electron-hole overlaps for all possible optical transitions in the InGaN QW region for various polarization degrees $\Delta P$, which corresponds to various built-in electric fields. Analyzing the five diagrams, it is observed that the absorption edge in the InGaN MQW region shifts to blue with the decrease of the internal electric field that is a well-known behavior for such a QW. In addition, a lot of non-zero electron-hole overlaps is observed at energies higher than the ground state transition energy.

Comparing the results of theoretical calculations with the energy of SE and CER transition, it has been concluded that the degree of polarization in the investigated $\mathrm{InGaN} \mathrm{QW}$ is close to $75 \%$. This reduction of piezoelectric field in this QW system can be attributed to Si doping in QW barriers. Much higher reduction of the built-in electric field is observed for strong optical pumping $(P>5 \mu \mathrm{J})$ for which the lasing from the structure is observed at $475 \mathrm{~nm}$ [see the blue line Fig. 1(a)]. The $25 \mathrm{~nm}$ shift between the SE and lasing is explained by the screening of built-in electric field by photogenerated carriers. However, it is worth noting that the built-in electric field in InGaN QW is not fully screened when the lasing from this structure is observed. This conclusion can be extracted from our calculations since the ground state transition in the fully screened InGaN QW is expected at $\sim 440 \mathrm{~nm}$ whereas the lasing from InGaN QW is observed at $475 \mathrm{~nm}$. This wavelength corresponds to the polarization degree of $\sim 50 \%$, see Fig. 1(c). It is worth noting that also other authors reported that lasing from polar InGaN QWs is expected/observed when the electric field related to polarization effects is partially screened [14, 32, 33].

In general, the phenomenon of electric field screening at lasing is to be expected for polar InGaN QWs since a high carrier density is necessary for lasing. On the other hand it should be much easier to obtain an inversion of carrier population in polar InGaN QW system (i.e., lower pumping intensity is expected) because of longer recombination time for InGaN QWs with strong built-in electric field. Unfortunately, the decay time of emission is strongly modified at room temperature by non-radiative centers and, therefore, 


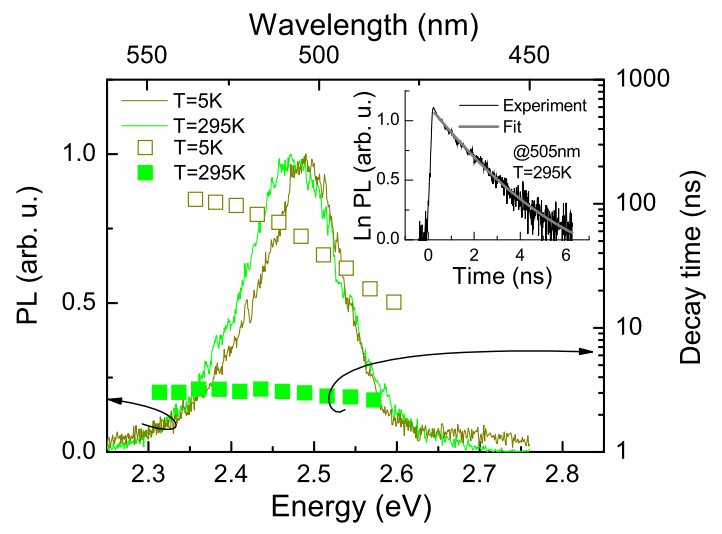

Fig. 3 Normalized photoluminescence spectra obtained at $5 \mathrm{~K}$ (dark green line) and $295 \mathrm{~K}$ (green line) just after the excitation impulse together with the decay time determined for various energies. The inset shows the decay of photoluminescence signal at $505 \mathrm{~nm}$ together with the fit by a single-exponential formula

a significant part of the pumping intensity is lost due to non-radiative recombination. Higher concentration of native point defects (i.e., the source of non-radiative recombination) is typical for epitaxial structures grown at lower temperatures (such growth conditions are often required to prevent the atom segregation and/or enhance the incorporation of some atoms) [34, 35]. The existence of significant concentration of non-radiative centers in our InGaN QW structures is confirmed by TRPL measurements. In addition, this measurements support the conclusion that the character of $\mathrm{SE}$ at room temperature is delocalized.

Figure 3 shows PL spectra just after the excitation impulse together with the decay time of PL $\left(\tau_{\mathrm{PL}}\right)$ determined for different energies of PL peak. The weak shift of PL peak with the temperature is the first evidence for carrier localization in this QW at low temperatures. Neglecting the temperature-induced changes in the built-in electric, which can be possible for this material system, the temperature shift of the fundamental QW transition in a homogeneous InGaN QW without localization phenomena should be similar to the shift of energy gap for $\mathrm{GaN}$, which equals $70 \mathrm{meV}$ when the temperature is increased from 5 to $295 \mathrm{~K}$ [25]. In our case it is only $25 \mathrm{meV}$ vs. $70 \mathrm{meV}$ since the low temperature PL corresponds to emission of localized excitons. Additional evidence for carrier localization at low temperatures is provided by the analysis of spectral dispersion of $\tau_{\mathrm{PL}}$. The $\tau_{\mathrm{PL}}$ has been obtained by fitting the experimental data by a single-exponential decay of PL signal $I(t)=I_{0} \exp \left(-t / \tau_{\mathrm{PL}}\right)$, where $t$ is the time and $I_{0}$ is the intensity of PL signal just after the excitation impulse. The $\tau_{\mathrm{PL}}$ depends on both the radiative $\left(\tau_{\mathrm{r}}\right)$ and the non-radiative $\left(\tau_{\mathrm{nr}}\right)$ recombination time according to Eq. (8)

$\frac{1}{\tau_{\mathrm{PL}}}=\frac{1}{\tau_{\mathrm{r}}}+\frac{1}{\tau_{\mathrm{nr}}}$
The radiative recombination time for free excitons in a QW results from the quantum confinement and the distribution of excitons in $k$-space [36]. The non-radiative recombination time is directly associated with the existence of point defects, which are the source of non-radiative recombination and whose activity depends on the temperature. The stronger non-radiative recombination means shorter $\tau_{\mathrm{nr}}$. According to Eq. (8) the shorter $\tau_{\mathrm{nr}}$ leads to a shorter $\tau_{\mathrm{PL}}$. At low temperatures the carrier localization suppresses the influence of non-radiative recombination on $\tau_{\mathrm{PL}}$ and leads to a spectral dispersion of $\tau_{\mathrm{PL}}$. This dispersion appears due to hopping of excitons between localizing potentials and their effective transfer to areas with larger localization energy and longer $\tau_{\mathrm{PL}}$ [37-39]. It means that the dispersion of $\tau_{\mathrm{PL}}$ can be treated as a fingerprint of exciton localization. Such a dispersion is observed for InGaN QW at low temperatures, see open squares in Fig. 3. It is worth noting that the localized character of PL is expected at low temperatures for this material system even for high quality QWs [40-42]. The importance of non-radiative recombination is difficult to discuss in this case since the mechanism of non-radiative recombination is suppressed at low temperatures, i.e., $\tau_{\mathrm{nr}} \gg \tau_{\mathrm{r}}$. With the temperature increase the spectral dispersion of $\tau_{\mathrm{PL}}$ disappears (this effect is associated mainly with a weaker contribution of localized exciton emission to PL peak at higher temperatures) and the $\tau_{\mathrm{PL}}$ decreases due to stronger influence of non-radiative recombination, i.e., the $\tau_{\mathrm{nr}}$ starts to be shorter than the $\tau_{\mathrm{r}}$. Finally no dispersion of $\tau_{\mathrm{PL}}$ is observed at room temperature, see solid squares in Fig. 3. Because of no dispersion of $\tau_{\mathrm{PL}}$ at room temperature the SE can be attributed to free exciton recombination, which is significantly influenced by non-radiative recombination associated with point defects existing in the InGaN QW region. However, it is not excluded that a small contribution of the recombination of localized excitons is still present at room temperature. The large concentration of point defects leads to lasing at larger pumping intensity or even no lasing from the structure (i.e., the threshold of pumping intensity increases with the rise of defect concentration).

In addition to point defects, there is other feature of InGaN QWs which also leads to difficulties with lasing at longer wavelengths. It is the inhomogeneity of the QW potential, which is associated with some fluctuations of QW width and indium concentration and is responsible for the broadening of SE [43].

It is worth noting that the large broadening of the fundamental transition leads to a broad gain spectrum. Such a gain can be profitable in many applications (e.g. tunable semiconductor optical fiber ring lasers [44] and distributed Bragg reflector lasers where the emission wavelength can be tuned by the passive part of the laser structure and for operating in broad spectral range such a laser needs the active part with a broad gain spectrum $[45,46])$. Unfortunately, the 
broad gain spectrum is not favorable in edge emitting InGaN QW lasers since the gain maximum can be shifted to blue in comparison to the SE peak position and, therefore, the lasing will be observed at shorter wavelength than the SE. In addition, the broadening of optical gain, which is related to QW inhomogeneities, decreases the gain value in the peak position. Also the built-in electric field is this factor which decreases the value of optical gain [14]. Finally to achieve a positive gain in inhomogeneous polar InGaN QWs much larger carrier concentration is required than this one needed for a perfectly homogeneous QW. It means a larger screening of built-in electric field at lasing conditions and, hence, larger blueshift of lasing in comparison to SE.

Because of above-mentioned arguments we believe that QW inhomogeneities (i.e., fluctuations of QW width and indium concentration) are one of the driving force for the blue shift between lasing and SE in the InGaN QW system. We expect that the reduction of QW inhomogeneities in the investigated $\mathrm{QW}$ should lead to a smaller shift between lasing and SE than this one, which is observed in the present sample. However, we would like to underline that QW inhomogeneities are not very high in the investigated sample. The single-exponential decay of PL at room temperature (see inset in Fig. 3) confirms this conclusions. For very inhomogeneous InGaN QWs we observed non-exponential decay of $\mathrm{PL}$ at room temperature that is evidence of carrier localization related to strong QW inhomogeneity. It has been shown theoretically that the non-exponential behavior of PL decay is a feature typical for very inhomogeneous QWs [24]. The observation of very nice single-exponential decay of PL at room temperature is an additional argument that the localized emission can be neglected in this sample at room temperature. At low temperatures we have observed some deviation from the single-exponential decay. This observation besides the dispersion of PL decay time confirms the carrier localization at low temperatures. It is worth noting that the accuracy of PL decay time determined at low temperature is much smaller than this one determined at room temperature due to the mentioned non-exponential behavior PL decay at low temperatures.

In order to analyze the issue of QW inhomogeneities in our system the fundamental transition has been calculated for a random InGaN QW [47] and compared with results obtained for homogeneous quantum well, which can be treated as the reference. In this approach the intensity of fundamental transition is calculated thousand times for the randomly generated QW with the Gaussian distribution of QW width and indium concentration. This distribution is given by Eq. (9):

$g\left(x, \mu, \sigma^{2}\right)=\frac{1}{\sigma \sqrt{2 \pi}} \exp \left(\frac{1}{2}\left(\frac{x-\mu}{\sigma}\right)^{2}\right)$,

where $x$ is the QW width (or indium concentration), $\mu$ is the mean value in this distribution and $\sigma^{2}$ is the standard de- viation of $\sigma$, i.e., the broadening of Gaussian distribution. The mean value in the Gaussian distribution corresponds to the average value of QW width and indium concentration whereas the broadening of Gaussian distribution is the magnitude of QW width and indium concentration fluctuations. To illustrate the results of calculations for randomly generated QWs, appropriate histograms of the intensity of QW transitions are constructed. Such histograms are made in the following way: The energy scale is divided for narrow ranges with the width of $25 \mathrm{meV}$ and the intensity of optical transitions are summed in the each spectral range. Next the whole histogram is divided by the number of randomly generated QWs (it is a normalization procedure). In a result the each bar in such a histogram is proportional to the probability of optical transition at the given energy. For more details on calculations of the histograms of optical transitions in inhomogeneous QWs see Refs. [47, 48].

As seen in Fig. 4, the built-in electric field strongly influences the fundamental transition (its intensity and energy distribution). The intensity of the fundamental transition in the inhomogeneous QW is distributed over broad spectral range while for a perfect QW this intensity is distributed over $\sim k T$ energy. In addition, the energy of fundamental transition is higher for inhomogeneous QWs than for homogeneous QWs, see the difference $(\delta E)$ in Fig. 5(a). This effect is observed since the electron-hole overlap integral strongly decreases with the energy redshift and increases with the energy blueshift. These shifts for inhomogeneous polar QWs are generated by fluctuations of QW width and indium concentration. However, the most important conclusion from Fig. 4 is that for inhomogeneous QWs the amplitude of fundamental transition in the peak position is much weaker than the electron-hole overlap integral for homogeneous QWs, see the direct comparison of the two quantities in Fig. 5(b). It means that in real InGaN QW systems (inhomogeneous QWs) a positive optical gain should be expected at higher carrier concentration than for homogeneous QWs.

Very similar relations between the SE, lasing, and CER spectra were observed for other InGaN QW structures emitting in the blue-green spectral range including InGaN QWs with the SE at $530 \mathrm{~nm}$ which were lasing at $480 \mathrm{~nm}$. Finally it has been concluded that InGaN QW structures dedicated for green lasers should have a high homogeneity of QW width and indium concentration as well as low concentration levels of non-radiative centers. In general, it is obvious that to obtain lasing from a QW it is necessary to reduce the number of non-radiative centers and to improve the homogeneity of QW potential by the reduction QW width fluctuations and alloy content fluctuations. However, these requirements are extremely important for polar InGaN QWs, which are dedicated for green lasers, because of high indium concentration, small QW width and large built-in electric field in this system. The recent progress in the growth of green 


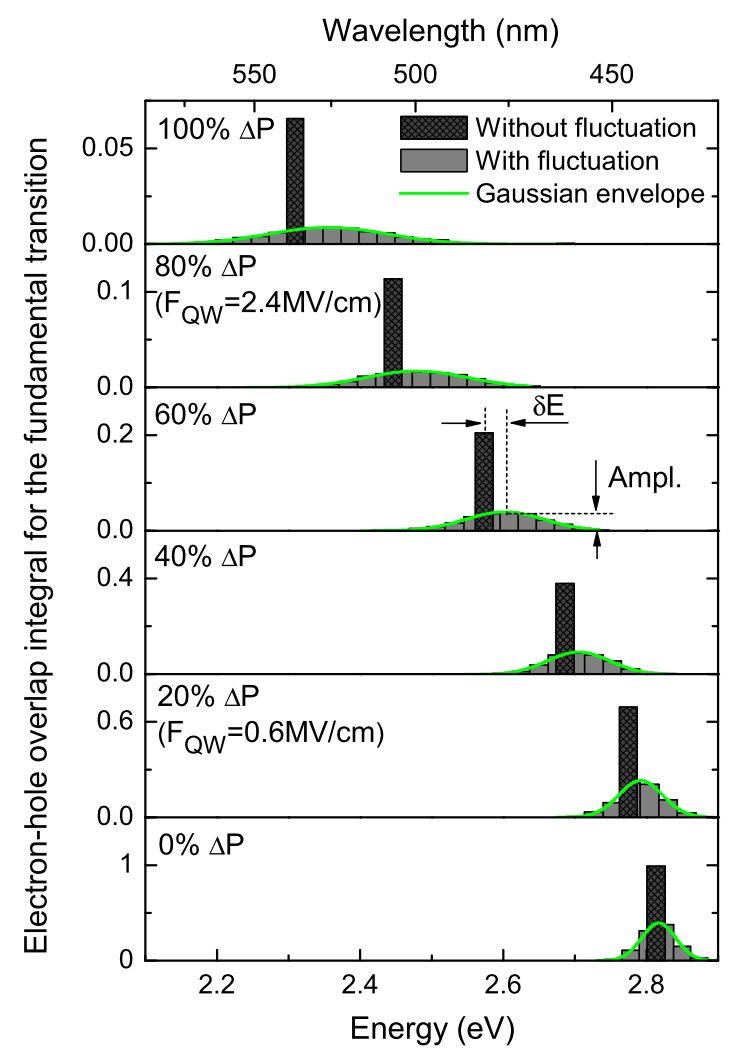

Fig. 4 The intensity of the fundamental transition in InGaN single QW without (dark bars with the pattern) and with the Gaussian fluctuation of QW width and indium concentration (gray bars). The fluctuation of

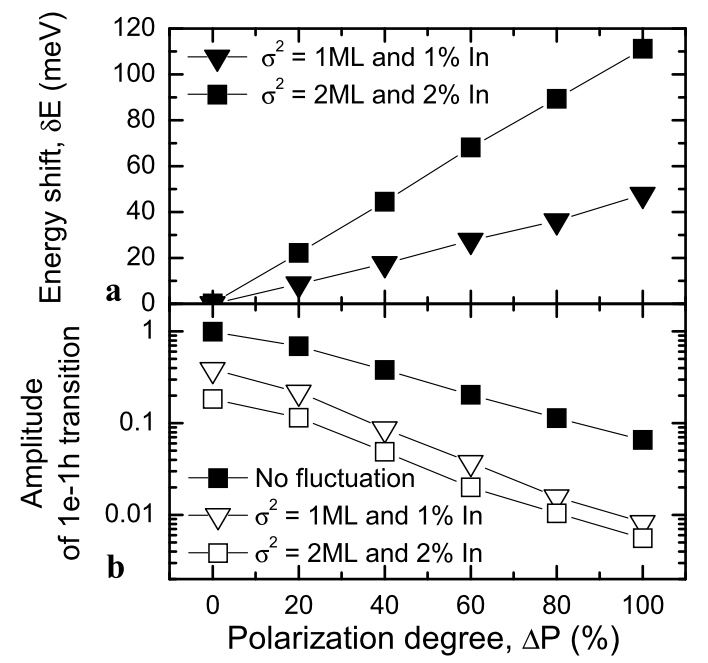

Fig. 5 (a) Energy shift, $\delta E$, between the ground state transition in homogeneous and inhomogeneous InGaN QW obtained for various polarization degrees $\Delta P$, which corresponds to various built-in electric fields. (b) Amplitude of the ground state transition in homogeneous (solid squares) and inhomogeneous (open squares and triangles) In$\mathrm{GaN}$ QW of various polarization degree $\Delta P$

lasers [2-4, 6-12, 49-52] have been obtained by using the metal organic vapor deposition while in this paper we report

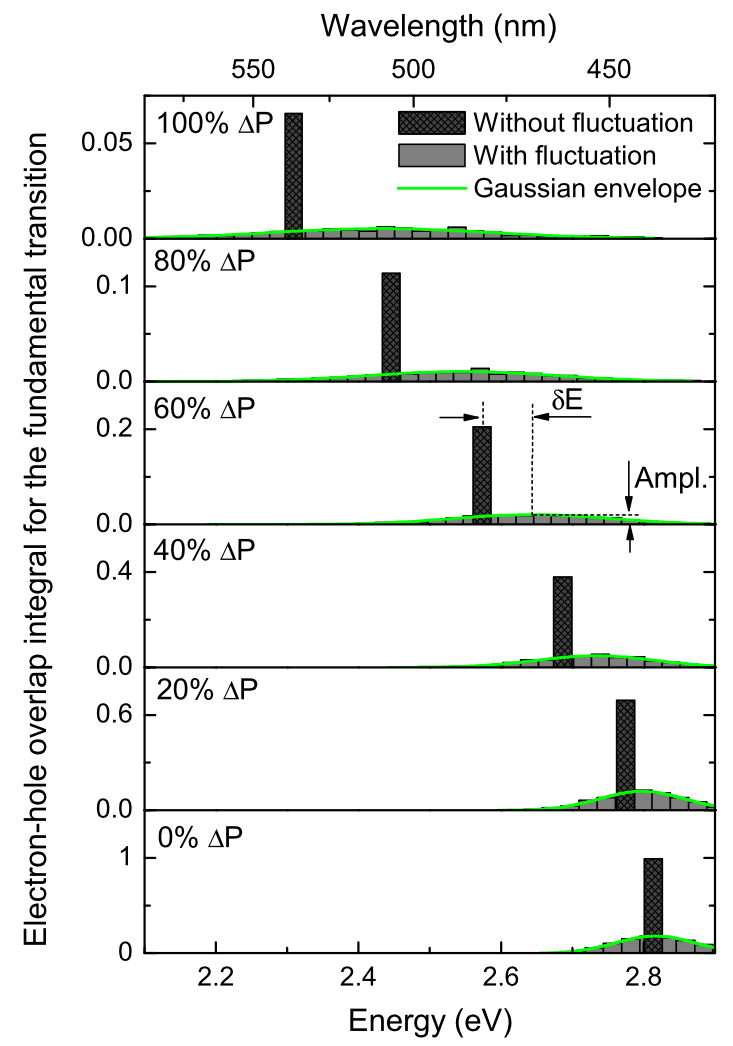

QW width and indium concentration $\left(\sigma^{2}\right)$ is assumed to be: 1 monolayer (ML) and $1 \%$ In (left panels) and 2MLs and $2 \%$ In (right panels)

results for structures grown by MBE. In general, different challenges appear for the two technologies in this field, but requirements for lasing from InGaN QWs should be very similar for structures grown by the two methods.

Finally it is worth noting that for careful treatment of the problem InGaN QW of various carrier concentrations the Schrödinger-Poisson equations have to be solved selfconsistently. Such calculations will modify quantitatively results obtained in this work but conclusions qualitatively will be the same. It is worth noting that the simple approach to calculate optical transitions and electron-hole overlaps for QW with various polarization degree instead of selfconsistent calculations for various carrier concentrations avoids problems with the computing time, boundary conditions, etc. In addition, this method allows to investigate theoretically changes in energies and electron-hole overlaps going from polar to non-polar QW system.

\section{Conclusions}

In conclusion, the large Stokes shift observed at room temperature between the SE and the optical transition in the absorption-like spectrum (i.e., CER spectrum) is not associ- 
ated with the carrier localization. This shift is observed because of the large built-in electric field in InGaN QW (i.e., the CER transition is a superposition of all optical transitions with non-zero electron-hole overlap integrals and, therefore, the energy of this transition does not correspond to the fundamental transition in InGaN QW). The lasing from this QW has been found at the wavelength of $475 \mathrm{~nm}$, whereas the SE was observed at $500 \mathrm{~nm}$. The $25 \mathrm{~nm}$ shift between the lasing and SE is observed because of a screening of the builtin electric field by photogenerated carriers. The large pumping intensity $(P>5 \mu \mathrm{J})$ is required for lasing in this system because of (i) a significant number of non-radiative centers and (ii) some inhomogeneities in QW potential which are induced by some fluctuations of QW width and indium concentration.

Acknowledgement This work was supported by the Polish Ministry of Science and Higher Education (Grant No. N N515 604439).

Open Access This article is distributed under the terms of the Creative Commons Attribution License which permits any use, distribution, and reproduction in any medium, provided the original author(s) and the source are credited.

\section{References}

1. S. Nakamura, G. Fasol, The Blue Laser Diode (Springer, Berlin, 1997)

2. T. Miyoshi, S. Masui, T. Okada, T. Yanamoto, T. Kozaki, S. Nagahana, T. Mukai, Appl. Phys. Express 2, 062201 (2009)

3. D. Queren, A. Avramescu, G. Brüderl, A. Breidenassel, M. Schillgalies, S. Lutgen, U. Strauß, Appl. Phys. Lett. 94, 081119 (2009)

4. Y. Enya, Y. Yoshizumi, T. Kyono, K. Akita, M. Ueno, M. Adachi, T. Sumitomo, S. Tokuyama, T. Ikegami, K. Katayama, T. Nakamura, Appl. Phys. Express 2, 082101 (2009)

5. C. Skierbiszewski, Z.R. Wasilewski, I. Grzegory, S. Porowski, J. Cryst. Growth 311, 1632 (2009)

6. T. Miyoshi, S. Masui, T. Okada, T. Yanamoto, T. Kozaki, S.-I. Nagahama, T. Mukai, Appl. Phys. Express 2, 062201 (2009)

7. Y. Yoshizumi, M. Adachi, Y. Enya, T. Kyono, S. Tokuyama, T. Sumitomo, K. Akita, T. Ikegami, M. Ueno, K. Katayama, T. Nakamura, Appl. Phys. Express 2, 092101 (2009)

8. D.S. Sizov, R. Bhat, J. Napierala, J. Xi, D.E. Allen, C.S. Gallinat, C. Zah, Opt. Lett. 34, 328 (2009)

9. A. Avramescu, T. Lermer, J. Müller, C. Eichler, G. Bruederl, M. Sabathil, S. Lutgen, U. Strauss, Appl. Phys. Express 3, 061003 (2011)

10. M. Adachi, Y. Yoshizumi, Y. Enya, T. Kyono, T. Sumitomo, S. Tokuyama, S. Takagi, K. Sumiyoshi, N. Saga, T. Ikegami, M. Ueno, K. Katayama Nakamura, Appl. Phys. Express 3, 121001 (2010)

11. Y.-D. Lin, S. Yamamoto, C.-Y. Huang, C.-L. Hsiung, F. Wu, K. Fujito, H. Ohta, J.S. Speck, S.P. DenBaars, S. Nakamura, Appl. Phys. Express 3, 082001 (2010)

12. M. Ueno, Y. Yoshizumi, Y. Enya, T. Kyono, M. Adachi, S. Takagi, S. Tokuyama, T. Sumitomo, K. Sumiyoshi, N. Saga, T. Ikegami, K. Katayama, T. Nakamura, J. Cryst. Growth 315, 258 (2011)

13. M. Siekacz, M. Sawicka, H. Turski, G. Cywiński, A. Khachapuridze, P. Perlin, T. Suski, M. Bockowski, J. Smalc-Koziorowska, M. Krysko, R. Kudrawiec, M. Syperek, J. Misiewicz, Z.R. Wasilewski, S. Porowski, C. Skierbiszewski, J. Appl. Phys. 110, $063110(2011)$
14. D. Sizov, R. Bhat, C.-E. Zah, J. Lightwave Technol. 30, 679 (2012)

15. F. Bernardini, V. Fiorentini, Phys. Rev. B 64, 085207 (2011)

16. O. Ambacher, J.A. Majewski, C. Miskys, A. Link, M. Hermann, M. Eickhoff, M. Stutzmann, F. Bernardini, V. Fiorentini, V. Tilak, B. Schaff, L.F. Eastman, J. Phys. Condens. Matter 14, 3399 (2002)

17. M. Motyka, R. Kudrawiec, J. Misiewicz, Phys. Status Solidi (a) 204, 354 (2007)

18. R. Kudrawiec, Phys. Status Solidi B 247, 1616 (2010)

19. R. Kudrawiec, J. Misiewicz, Rev. Sci. Instrum. 80, 096103 (2009)

20. B. Damilano, N. Grandjean, J. Massies, L. Siozade, J. Leymarie, Appl. Phys. Lett. 77, 1268 (2000)

21. R. Kudrawiec, G. Sęk, K. Ryczko, J. Misiewicz, P. Sundgren, C. Asplund, M. Hammar, Solid State Commun. 127, 613 (2003)

22. K. Kazlauskas, G. Tamulaitis, J. Mickevièius, E. Kuokštis, A. Žukauskas, Y.-C. Cheng, H.-C. Wang, C.-F. Huang, C.C. Yang, J. Appl. Phys. 97, 013525 (2005)

23. R. Kudrawiec, G. Sek, J. Misiewicz, F. Ishikawa, A. Trampert, K.H. Ploog, Appl. Phys. Lett. 94, 011907 (2009)

24. M. Gladysiewicz, R. Kudrawiec, Phys. Status Solidi (a) 209, 752 (2012)

25. I. Vurgaftman, J.R. Meyer, J. Appl. Phys. 94, 3675 (2003) and references therein

26. R. Kudrawiec, M. Siekacz, M. Kryśko, G. Cywiński, J. Misiewicz, C. Skierbiszewski, J. Appl. Phys. 106, 113517 (2009)

27. S.L. Chuang, C.S. Chang, Phys. Rev. B 54, 2491 (2006)

28. M. Leroux, N. Grandjean, M. Laugt, J. Massies, B. Gil, P. Lefebvre, P. Bigenwald, Phys. Rev. B 58, R13371 (1998)

29. E. Oh, C. Sone, O. Nam, H. Park, Y. Park, Appl. Phys. Lett. 76, $3242(2000)$

30. M.-Y. Ryu, P.W. Yu, E.-j. Shin, J.I. Lee, S.K. Yu, E. Oh, O.H. Nam, C.S. Sone, Y.J. Park, J. Appl. Phys. 89, 634 (2001)

31. C.K. Choi, Y.H. Kwon, B.D. Little, G.H. Gainer, J.J. Song, Y.C. Chang, S. Keller, U.K. Mishra, S.P. DenBaars, Phys. Rev. B 64, 245339 (2001)

32. G.E. Dialynas, G. Deligeorgis, M. Zervos, N.T. Pelekanos, J. Appl. Phys. 104, 113101 (2008)

33. U. Strauss, A. Avramescu, T. Lermer, D. Queren, A. GomezIglesias, C. Eichler, J. Muller, G. Bruderl, S. Lutgen, Phys. Status Solidi (b) 248, 652 (2011)

34. J.S. Harris, R. Kudrawiec, H.B. Yuen, S.R. Bank, H.P. Bae, M.A. Wistey, D. Jackrel, E.R. Pickett, T. Sarmiento, L.L. Goddard, V. Lordi, T. Gugov, Phys. Status Solidi (b) 244, 2707 (2007)

35. J.Y. Tsao, Materials Fundamentals of Molecular Beam Epitaxy (Academic Press, San Diego, 1993)

36. L.C. Andreani, Solid State Commun. 77, 641 (1991)

37. S.D. Baranovskii, R. Eichmann, P. Thomas, Phys. Rev. B 58, 13081 (1998)

38. Y.-C. Lu, C.-Y. Chen, H.-C. Wang, C.C. Yang, Y.-C. Cheng, J. Appl. Phys. 101, 063511 (2007)

39. M. Baranowski, R. Kudrawiec, M. Latkowska, M. Syperek, J. Misiewicz, J.A. Gupta, Appl. Phys. Lett. 100, 202105 (2012)

40. J.H. Na, R.A. Taylor, K.H. Lee, T. Wang, A. Tahraoui, P. Parbrook, A.M. Fox, S.N. Yi, Y.S. Park, J.W. Choi, J.S. Lee, Appl. Phys. Lett. 89, 253120 (2006)

41. Y.-C. Cheng, E.-C. Lin, C.-M. Wu, C.C. Yang, J.-R. Yang, A. Rosenauer, K.-J. Ma, S.-C. Shi, L.C. Chen, C.C. Pan, J.I. Chyi, Appl. Phys. Lett. 84, 2506 (2004)

42. D. Watson-Parris, M.J. Godfrey, P. Dawson, R.A. Oliver, M.J. Galtrey, M.J. Kappers, C.J. Humphreys, Phys. Rev. B 83, 115321 (2001)

43. M. Gladysiewicz, R. Kudrawiec, Phys. Status Solidi (c) 9, 830 (2012)

44. S. Oshiba, K. Nagai, M. Kawahara, A. Watanabe, Y. Kawai, Appl. Phys. Lett. 55, 2383 (1989) 
45. J.-P. Reithmaier, A. Forchel, IEEE J. Sel. Top. Quantum Electron. 8, 1035 (2002)

46. M. Gioannini, I. Montrosset, IEE Proc. Optoelectron. 148, 13 (2001)

47. M. Gladysiewicz, R. Kudrawiec, J. Phys. Condens. Matter 22, 485801 (2010)

48. M. Gladysiewicz, R. Kudrawiec, Phys. Status Solidi (c) 9, 1007 (2011)

49. D. Sizov, R. Bhat, K. Song, D. Allen, B. Paddock, S. Coleman, L.C. Hughes, C. Zah, Appl. Phys. Express 4, 102103 (2011)

50. A. Tyagi, R.M. Farrell, K.M. Kelchner, C.Y. Huang, P.S. Hsu, D.A. Haeger, M.T. Hardy, C. Holder, K. Fujito, D.A. Cohen, H. Ohta, J.S. Speck, S.P. DenBaars, S. Nakamura, Appl. Phys. Express 3, $011002(2010)$
51. S. Takagi, Y. Enya, T. Kyono, M. Adachi, Y. Yoshizumi, T. Sumitomo, Y. Yamanaka, T. Kumano, S. Tokuyama, K. Sumiyoshi, N. Saga, M. Ueno, K. Katayama, T. Ikegami, T. Nakamura, K. Yanashima, H. Nakajima, K. Tasai, K. Naganuma, N. Fuutagawa, Y. Takiguchi, T. Hamaguchi, M. Ikeda, Appl. Phys. Express 5, 082102 (2012)

52. K. Yanashima, H. Nakajima, K. Tasai, K. Naganuma, N. Fuutagawa, Y. Takiguchi, T. Hamaguchi, M. Ikeda, Y. Enya, S. Takagi, M. Adachi, T. Kyono, Y. Yoshizumi, T. Sumitomo, Y. Yamanaka, T. Kumano, S. Tokuyama, K. Sumiyoshi, N. Saga, M. Ueno, K. Katayama, T. Ikegami, T. Nakamura, Appl. Phys. Express 5, 082103 (2012) 